\title{
FACTORES SOCIO-CULTURALES DETERMINANTES EN LA ELECCIÓN DE UN TRATAMIENTO ODONTOLÓGICO
}

\section{FACTORS SOCIO-CULTURAL DETERMINANTS IN THE ELECTION OF AN ODONTOLOGICAL TREATMENT}

\author{
Valencia Berrezueta Marcela ${ }^{1 *}$, Urgilés Vintimilla Silvia ${ }^{2}$, Cuenca León Katherine ${ }^{3}$ \\ ${ }^{1}$ Carrera de Odontología Universidad Católica de Cuenca. Ecuador \\ ${ }^{2}$ Carrera de Odontología Universidad Católica de Cuenca. Ecuador ${ }^{3}$ Bioquímica farmacéutica, Magister en Gerencia en \\ Salud para el Desarrollo Local, Docente de la Universidad Católica de Cuenca. Ecuador \\ *solemarceva@gmail.com
}

\begin{abstract}
Resumen
OBJETIVO: Determinar los factores socio-culturales determinantes en la elección de un tratamiento odontológico en la parroquia Yanuncay y Tarqui del cantón Cuenca. MÉTODOS: Se realizó un estudio de tipo cuantitativo, diseño descriptivo, con un universo de 16.820 personas como muestra 374 de parroquia urbana y 367 de parroquia rural seleccionadas a través de muestreo estratificado por afijación óptima. Como variable dependiente se midió el grado de satisfacción de experiencia odontológica previa a la atención dental, el nivel socioeconómico mediante la encuesta INEC, y los hábitos de salud bucal mediante una encuesta elaborada para este estudio. RESULTADOS: La relación entre el nivel socioeconómico y la frecuencia de visitas al servicio odontológico presentó significancia estadística en ambas localidades (Yanuncay $\mathrm{p}=0.02$ y Tarqui p <0.001). El grado de satisfacción está relacionado significativamente con la experiencia odontológica en ambas parroquias con un valor de $\mathrm{p}<0.001$. Los hábitos que mostraron relación significativa con el uso de servicios odontológicos fueron: tener cepillo dental y visita al odontólogo, con $\mathrm{p}=0.005$ y $\mathrm{p}=0.029$ respectivamente. En la zona urbana únicamente el cambio de cepillo en diferentes periodos de tiempo presentó un valor de $p=0.01$. CONCLUSIONES: Los pobladores de las parroquias Tarqui y Yanuncay asisten a los servicios odontológicos de acuerdo a su nivel socioeconómico, grado de satisfacción del usuario y hábitos más frecuentes en salud oral de los pobladores.
\end{abstract}

Palabras clave: Necesidades y demandas en servicios de salud, Cultura, Hábitos y Satisfacción personal.

\begin{abstract}
OBJECTIVE: To determine the socio-cultural factors determining the choice of a dental treatment in the parish of Yanuncay and Tarqui in the canton of Cuenca. METHODS: A quantitative, descriptive design study was carried out with a population of 16,820 people, as sample 374 from an urban parish and 367 from a rural parish, selected through stratified sampling by optimal allocation. As dependent variable, the degree of satisfaction of dental experience prior to dental care, socioeconomic level through the INEC survey, and oral health habits were measured through a survey developed for this study. RESULTS: The relationship between the socioeconomic level and the frequency of visits to the dental service presented statistical significance in both localities (Yanuncay $p=0.02$ and Tarqui $p<0.001$ ). The degree of satisfaction is significantly related to the dental experience in both parishes with a value of $p<0.001$. The habits that showed a significant relationship with the use of dental services were: having a dental brush and visiting the dentist, with $p=0.005$ and $p=0.029$ respectively. In the urban area only change of brush in different periods of time presented a value of $p=0.01$. CONCLUSIONS: The inhabitants of the Tarqui and Yanuncay parishes attend dental services according to their socioeconomic level, degree of satisfaction of the user and more frequent habits in oral health of the settlers.
\end{abstract}

Key words: Needs and demands in health services, Culture, Habits and Personal satisfaction.

\section{INTRODUCCIÓN.}

La Odontología es una disciplina multidisciplinaria que aporta a la prevención y tratamiento de las enfermedades orales, aportando al adelanto de la sociedad por medio de la investigación, encontrándose distintos problemas tanto socia- les como culturales, como de la conducta humana que van a influir en la salud bucal de la población. ${ }^{1-4}$ Se han realizado a lo largo de la historia numerosos estudios que se han centrado en explicar los factores que influyen en la utilización de servicios dentales, resultando ser aspectos como: Edad, se- 
Tabla 1. Nivel Socio Económico.

\begin{tabular}{lcccccccccc}
\hline & \multicolumn{4}{c}{ YANUNCAY } & \multicolumn{4}{c}{ TARQUI } & \multicolumn{2}{c}{ Chi cuadrado } \\
& \multicolumn{2}{c}{ ACUDE } & \multicolumn{2}{c}{ NO ACUDE } & \multicolumn{2}{c}{ ACUDE } & \multicolumn{2}{c}{ NO ACUDE } & Sig. Yanuncay & Sig. Tarqui \\
& $\mathrm{n}$ & $\%$ & $\mathrm{n}$ & $\%$ & $\mathrm{n}$ & $\%$ & $\mathrm{n}$ & $\%$ & & \\
\hline Alto & 49 & 14.4 & 8 & 36.36 & 228 & 64.40 & 5 & 25 & & \\
Medio & 41 & 11.88 & 3 & 13.63 & 116 & 32.76 & 11 & 55 & 0.02 & $\mathrm{p}<0.01$ \\
Bajo & 255 & 73.91 & 11 & 50 & 10 & 2.82 & 4 & 20 & & \\
TOTAL & 345 & 100 & 22 & 100 & 354 & 100 & 20 & 100 & & \\
\hline
\end{tabular}

Tabla 2. Grado de satisfacción con la experiencia odontológica.

\begin{tabular}{lccccc}
\hline & \multicolumn{2}{c}{ YANUNCAY } & \multicolumn{2}{c}{ TARQUI } & Chi cuadrado \\
& $\mathrm{n}$ & $\%$ & $\mathrm{n}$ & $\%$ & Sig. \\
\hline Satisfecho & 306 & 81.82 & 180 & 49.05 & $\mathrm{P}<0.001$ \\
Insatisfecho & 68 & 18.18 & 187 & 50.95 & 100 \\
TOTAL & 374 & 100 & 364 & & 100 \\
\hline
\end{tabular}

xo, educación, ocupación, ingreso, estatus socioeconómico, ubicación geográfica, estilos de vida, experiencias previas de atención y presencia o ausencia de síntomas u otras. ${ }^{5} \mathrm{Al}$ identificar una gran cantidad de motivos que parecen estar asociados con la decisión de buscar ayuda profesional, se han adoptado modelos explicativos que pretenden aclarar las relaciones entre las características individuales, sociales, económicas y culturales que llevan a buscar ayuda profesional. Sin embargo, ha sido necesario que los investigadores establezcan algunos criterios comunes para que sus trabajos sobre este tema puedan ser comprobados o incluso confirmados en otros países. ${ }^{6,7}$

Se requiere precisar la cantidad de servicios de salud que los individuos desean consumir a costos específicos. La idea en cuanto a mercados de salud nos ha llevado a pensar que la alimentación, los hábitos, la higiene y la búsqueda de atención son asuntos que obedecen al comportamiento individual de cada ser humano y que la prestación de servicios de salud oral es un asunto técnico, cuando la realidad conlleva a trabajar en conjunto para alcanzar las metas sobre salud bucal ya que los factores que la condicionan son variables y por ende modificables. ${ }^{8,9}$

Como odontólogos debemos brindar un servicio de salud de calidad y eficiente tratando de buscar equidad y por ende aportando a cumplir los objetivos de salud bucal, además desempeña parte del proceso salud enfermedad es decir guarda relación con los determinantes sociales y culturales en la población. ${ }^{10}$

El objetivo de la presente investigación fue: determinar los factores socio-culturales determinantes en la elección de un tratamiento odontológico en la parroquia urbana Yanuncay y la parroquia rural Tarqui del cantón Cuenca-Ecuador.

\section{MATERIALES Y MÉTODOS.}

Se realizó un estudio de tipo cuantitativo de diseño descriptivo, el universo de estudio estuvo constituido por 16.820 personas, en edades de 25 a 40 años, la muestra analizada fue de 374 personas de la zona urbana y 367 de la zona rural. Se aplicaron encuestas a las personas que querían formar parte del estudio y cumplían con los criterios de inclusión. Para el análisis estadístico se utilizó el software de acceso libre Open Epi y de esta manera se calculó el Chi-cuadrado. Todos los individuos en estudio firmaron un consentimiento informado para la participación en el mismo.

\section{RESULTADOS.}

La presente investigación, realizada en las parroquias de Yanuncay y Tarqui, muestran que la gran mayoría de encuestados que acceden a los servicios de salud odontológica en el área urbana son de estrato socio económico bajo y en la parroquia rural son de nivel alto (Tabla 1) El grado de satisfacción está relacionado significativamente con la experiencia odontológica en ambas parroquias con un valor de $\mathrm{p}<0.001$ (Tabla 2). Los hábitos que mostraron relación significativa con el uso de servicios odontológicos fueron: tener cepillo dental y visita al odontólogo, con $\mathrm{p}=0.005$ y $\mathrm{p}=0.029$ respectivamente. En la zona urbana únicamente cambio de cepillo en diferentes periodos de tiempo presentó un valor de $\mathrm{p}=0.01$ (Tabla 3 ).

\section{DISCUSIÓN.}

La presente investigación, realizada en las parroquias de Yanuncay y Tarqui, muestran que la gran mayoría de encuestados que acceden a los servicios de salud odontológica en el 
Tabla 3. Relación entra los hábitos más frecuentes en salud oral y su relación con el uso de servicios odontológicos.

\begin{tabular}{|c|c|c|c|c|c|c|c|c|c|c|}
\hline & \multicolumn{4}{|c|}{$\begin{array}{c}\text { Parroquia Yanuncay } \\
\text { (Urbana) }\end{array}$} & \multicolumn{4}{|c|}{$\begin{array}{c}\text { Parroquia Tarqui } \\
\text { (Rural) }\end{array}$} & \multicolumn{2}{|c|}{ Chi cuadrado } \\
\hline & \multicolumn{2}{|c|}{ Acude } & \multicolumn{2}{|c|}{ No acude } & \multicolumn{2}{|c|}{ Acude } & \multicolumn{2}{|c|}{ No acude } & $\begin{array}{c}\text { Sig. } \\
\text { Yanuncay }\end{array}$ & Sig. Tarqu \\
\hline Fuma de 0 a 2 cigarros al día. & 127 & 33.95 & 9 & 2.41 & 191 & 52,04 & 16 & 4,35 & \multirow{3}{*}{0.70} & \multirow{3}{*}{0.27} \\
\hline Fuma media caja de cigarros al día. & 182 & 48.66 & 9 & 2.41 & 121 & 31,97 & 5 & 1,36 & & \\
\hline Fuma más de una caja de cigarros al día. & 45 & 12.03 & 2 & 0.53 & 33 & 8,99 & 1 & 0,27 & & \\
\hline Se muerde las uñas. & 142 & 37.96 & 11 & 2.94 & 166 & 45.23 & 13 & 3.54 & \multirow{2}{*}{0.2011} & \multirow{2}{*}{0.32} \\
\hline No se muerde las uñas. & 212 & 56.68 & 9 & 2.41 & 179 & 48.77 & 9 & 2.45 & & \\
\hline Si tiene cepillo dental en casa. & 325 & 86.90 & 29 & 7.75 & 341 & 92.91 & 19 & 5.17 & \multirow{2}{*}{0.73} & \multirow{2}{*}{0.005} \\
\hline No tiene cepillo dental en casa. & 18 & 4.81 & 2 & 0.53 & 4 & 1.08 & 3 & 0.81 & & \\
\hline Comparte su cepillo dental en casa. & 16 & 4.28 & 1 & 0.28 & 39 & 10.62 & 4 & 1.08 & \multirow{2}{*}{0.84} & \multirow{2}{*}{0.349} \\
\hline No comparte su cepillo dental en casa. & 338 & 90.37 & 19 & 5.08 & 306 & 83.37 & 18 & 4.90 & & \\
\hline Le sangran las encías al momento del cepillado. & 155 & 41.44 & 7 & 1.87 & 211 & 57.49 & 18 & 4.90 & \multirow{2}{*}{0.45} & \multirow{2}{*}{0.05} \\
\hline No le sangran las encías al momento del cepillado. & 199 & 53.21 & 13 & 3.48 & 134 & 36.51 & 4 & 1.08 & & \\
\hline Espera de 1 a 5 horas para ir al odontólogo por dolor dental. & 186 & 49.73 & 12 & 3.21 & 64 & 17.43 & 1 & 0.27 & & \\
\hline Espera de 1 a 2 días para ir al odontólogo por dolor dental. & 132 & 35.29 & 8 & 2.14 & 130 & 35.42 & 4 & $1-08$ & & \\
\hline Espera de 2 a 5 días para ir al odontólogo por dolor dental. & 36 & 9.63 & 0 & 0.00 & 113 & 30.79 & 3 & 0.81 & 0.32 & 0,83 \\
\hline Cree que debe ir al odontólogo cuando siente dolor dental. & 86 & 22.99 & 2 & 0.53 & 81 & 22.07 & 10 & 2.72 & & \\
\hline Cree que debe ir al odontólogo 2 veces al año. & 94 & 25.13 & 6 & 1.60 & 167 & 45.50 & 5 & 1.36 & 0.33 & 0,029 \\
\hline Cepilla sus dientes 1 vez al día & 129 & 34.49 & 9 & 2.41 & 138 & 37.60 & 10 & 2.12 & & \\
\hline Cepilla sus dientes 3 veces al día. & 156 & 41.71 & 7 & 1.87 & 204 & 55.58 & 8 & 2.17 & 0.69 & N.A \\
\hline No cepilla sus dientes. & 69 & 18.44 & 4 & 1.07 & 3 & 0.81 & 4 & 1.08 & & \\
\hline Cambia su cepillo dental cada de 3 a 6 meses. & 120 & 32.09 & 5 & 1.37 & 105 & 28.61 & 5 & 1.36 & & \\
\hline Cambia su cepillo dental una vez al año. & 191 & 51.07 & 8 & 2.14 & 193 & 52.58 & 11 & 2.99 & 0.01 & 0.20 \\
\hline Cambia su cepillo dental cada dos años. & 43 & 11.50 & 7 & 1.87 & 47 & 12.80 & 6 & 1.63 & & \\
\hline
\end{tabular}

área urbana son de estrato socio económico bajo y en la parroquia rural son de nivel alto (Tabla 1) El grado de satisfacción está relacionado significativamente con la experiencia odontológica en ambas parroquias con un valor de $\mathrm{p}<0.001$ (Tabla 2). Los hábitos que mostraron relación significativa con el uso de servicios odontológicos fueron: tener cepillo dental y visita al odontólogo, con $\mathrm{p}=0.005$ y $\mathrm{p}=0.029$ respectivamente. En la zona urbana únicamente cambio de cepillo en diferentes periodos de tiempo presentó un valor de $\mathrm{p}=0.01$ (Tabla 3). La presente investigación realizada en las parroquias Yanuncay (urbana) y Tarqui (rural) del cantón Cuenca, presentó una relación positiva entre el grado de satisfacción y la experiencia odontológica, ya que fue estadísticamente significativa en ambos lugares con un valor de $\mathrm{p}<0.001$; esta investigación coincide con el trabajo de Fernández $\mathrm{C} \&$ cols en Chile en el 2011, el cual reporta que, a menor nivel de satisfacción con los servicios odontológicos más grave es la enfermedad oral. Al analizar con detalle los factores que determinaran el grado de satisfacción del encuestado se encontró que el tiempo que esperan los pacientes por atención odontológica es muy demorado especialmente en el sector público lo cual es contradictorio puesto que dentro de los beneficios del plan 
de salud del Ecuador se garantiza acceder al servicio de salud de inmediato evitando de esta manera poner en riesgo su salud, seguido de que en el sector privado los honorarios cobrados son muy altos agregando a esto, el miedo y la ansiedad que generan los servicios dentales, se establece, por tanto, como el punto de partida para evaluar la eficacia y calidad del servicio junto a la satisfacción generada por este. López J y cols, en el 2013 realizaron estudios en mujeres de 55 años de estratos económicos bajos donde la valoración del servicio dental fue positiva debido a la atención oportuna que recibieron ante sus problemas dentales, al profundizar más las entrevistas realizadas encontraron experiencias subjetivas propias que no están al alcance del talento humano del servicio odontológico. Martínez J y cols, en el 2014 sugieren que se debe prestar atención a la dinámica familiar y a la igualdad de género para acceder a las redes de apoyo social y por ende salud bucal. La presente investigación, realizada en las parroquias de Yanuncay y Tarqui, muestran que la gran mayoría de encuestados que acceden a los servicios de salud odontológica en el área urbana son de estrato socio económico bajo y en la parroquia rural son de nivel alto $\mathrm{Al}$ analizar el nivel socio-económico y el acceso a los servicios de salud odontológico en la parroquia Yanuncay en su mayoría fueron procedentes de nivel socioeconómico bajo con un $73.91 \%$ de la población y una minoría del $11.88 \%$ Medio, esto puede estar definido por el acceso total de los encuestados y sus familiares a todos los servicios básicos, especialmente a la red pública de alcantarillado y agua potable coincidiendo con los resultados del INEC 2011, el $11.2 \%$ de la población del Ecuador es de este nivel socio-económico siendo la provincia del Azuay especialmente en el Cantón Cuenca con menos índice de pobreza, la parroquia Yanuncay se encuentra en un lugar estratégico del catón siendo una zona comercial y con mayor acceso de salud pública, sin embargo en el sector rural hay predominio de una mayor asistencia del sector rural clase alta a servicios odontológicos, seguidos del medio. Paredes S y cols en el 2015 realizaron un estudio en niños de escuela en los que encontraron que tan solo 1 de cada 4 niños asistieron al centro de salud en los últimos 12 meses, la falta de dinero fue la principal causa acompañado de la ubicación geográfica en la que vive. Con respecto a los hábitos encontrados en la parroquia Yanuncay se encontró que el $41.71 \%$ de la población que asiste al odontólogo cepilla tres veces al día sus dientes coincidiendo con el estudio de Reyes $\mathrm{J}$ y cols que reportó un $45.83 \%$. En la parroquia Tarqui a pesar de ser sector rural el $55.58 \%$ de la población que asiste al odontólogo cepilla sus dietes tres veces al día siendo mayor al sector urbano y al estudio de Reyes J y cols. El $46.52 \%$ cree que debe ir al doctor una vez al año y el $22.99 \%$ cada vez que siente dolor dental probablemente por la experiencia negativa experimentada difiriendo notablemente al reportado por Carrasco y cols que reportaron que el $80 \%$ de los encuestados acudieron por dolor al dentista. En la parroquia Tarqui el $45.50 \%$ cree que debe ir al odontólogo dos veces al año y tan sólo el $22.07 \%$ cuando siente dolor. La presente investigación permite concluir que los pobladores de la parroquia Yanuncay asisten a los servicios odontológicos de acuerdo a su nivel socioeconómico, a las experiencias previas con el odontólogo (grado de satisfacción del usuario) y más concretamente a la cultura en salud oral de los pobladores. Si bien los resultados de este estudio no son generalizables a todos los habitantes de la parroquia Yanuncay, muestran la realidad de la salud oral de un grupo de pobladores.

Conflicto de intereses y financiamiento. Los autores declaran no tener conflicto de intereses, haber cumplido con los requisitos de autoría y haber autofinanciado este artículo.

\section{Referencias}

1 Paredes S, Juárerez P, Mosqueda A. Factores que influyen en la asistencia de los prescolares al servicio dental público en Acapulco, Guerrero, México. Rev. Odon. Méx.19 (1): 8-14 [Internet] 2015 [Citado en: 19/08/2016] Documento disponible

2 Martínez J, Capote J, Bermúdez G, Martínez Y. Determinantes sociales del estado de salud oral en el contexto actual. Rev. Scielo 12 (4): 562-569 [Internet] 2014 [Citado en: 19/08/2016] Documento disponible

3 Agudelo A, López F, Alzate S, López C, Espinoza E, Posada A, Menezes E. Salud bucal y género en relación a la población adulta mayor atendida en la red hospitalaria pública de Medellín: Los puntos de vista del personal de salud. Rev Scielo Colombia. 58:68 [Internet] 2015 [Citado en: 19/08/2016] Documento disponible

4 López J, Pilataxi S, Velásquez A, López M, Martínez C, Ágüelo A. Determinantes de la satisfacción odontológica en un grupo de pacientes atendidos en la clínica del adulto de la facultad odontológica de Antóquia Rev. Gerenc. Polit. Salud, Bogotá Colombia 12 (24) 209-225 [Internet] (2013) [Citado 19/08/2015]. Documento disponible

5 Flores L. López C. Factores que influyen en la utilización de los servicios odontológicos. Revisión de la literatura. Rev. Asociación Mexicana. 59 (3): 100-109 [Internet] 2002 [Citado en: 20/08/2016].Documento disponible

6 Fernández C, Núñez F, Zanzana D. Determinantes de la salud oral en población de 12 años Maule - Chile. Rev. Clin. Periodoncia Implantol. Rehabil. Oral 4(3): 117-121 [Internet] 2011 [Citado en: 21/08/2016] Documento disponible

7 Carrasco M, Landuro A, Orejuela F. Factores asociados a la utilización de servicios en una unidad clínica odontológica peruana. Rev. Estomatol Herediana 25 (1): 27-35 [Internet] 2015 [Citado en: [20/08/2016] Documento disponible

8 INEC: [Citado en: 21/08/2016] Documento disponible

9 Villavicencio E. El tamaño muestral en tesis de post grado. ¿Cuántas personas debo encuestar? (2011) [Citado en: 01/09/2016] Documento disponible

10 Reyes J, Rodríguez L, Paz R. Determinación de la salud bucal en personas mayores institucionalizadas residentes 
en Lima, Perú. Rev. KIRU 10 (2): 140-4 [Internet (2013)

[Citado en: 01/09/2016] Documento disponible

Recibido: 16 de Enero de 2017

Aceptado: 23 de Marzo de 2017 
